\title{
On an Attempt to Study the Inter-relationships amongst the Challenges Faced by Disabled Women and Sufferers of Domestic and Workplace Violence
}

\author{
Mahua Dutta \\ Ex-Senior Research \\ Fellow RAC, DRDO \\ Delhi, India
}

\author{
Neeta Panchmukh \\ MIT School of Education \& \\ Research, MIT Art, \\ Design \& Technological \\ University \\ Pune, India
}

\author{
Veena Aggarwal \\ Recventures Education \\ Services Private Limited \\ Delhi, India
}

\author{
Anu Aggarwal \\ NAWADCO, \\ Delhi, India
}

\begin{abstract}
Persons with disabilities are the world's largest minority, representing $15 \%$ of the global population. $80 \%$ of this population live in developing countries. In a similar sense, there are sufferers of domestic violence, a majority of them being women. This article therefore, explores the various challenges faced by disabled persons and sufferers of domestic and workplace violence. It thereafter, studies the possible inter-relationships amongst them using ISM methodology.
\end{abstract}

\section{Keywords}

Domestic violence ; ISM methodology ; Disabled women

\section{INTRODUCTION}

The United Nations Declaration on the Rights of Disabled Persons described the term 'disabled person' as any person unable to ensure by himself or herself, wholly or partly, the necessities of a normal individual and/or social life, as a result of deficiency, either congenital or not, in his or her physical or mental capabilities. The term disability therefore in research terminology encompasses physical, sensory or mental impairments, or a combination of impairments which includes physical disability resulting from injury (eg. spinal cord injury, amputation), chronic disease (eg. multiple sclerosis, rheumatoid arthritis), or congenital conditions (eg. cerebral palsy, muscular dystrophy), sensory impairments consisting of hearing or visual impairments and mental impairments comprising developmental conditions (eg. intellectual disability), cognitive impairment (eg. traumatic brain injury), or psychiatric disability [1-12]. Many agencies reported that the women who accessed their services had more than one type of disability. Psychiatric disability was the most prevalent disability reported, accounting for 55 per cent of the women. Physical disabilities accounted for an estimated 32 per cent of women, 30 per cent were identified as having an intellectual disability, 16 per cent a neurological disability and 10 per cent had sensory disabilities.

Family and domestic violence against women is acknowledged as a pervasive and serious problem. It is a violation of trust and an abuse of power in a relationship where a woman should have the right to absolute safety. As per Australian public health association, a comprehensive definition of domestic violence includes examples of abusive behavior such as physical abuse, verbal abuse, economic abuse, social abuse, emotional and psychological abuse which cause pain and injury ; denial of sleep; warmth or nutrition ; denial of needed medical care ; sexual assault ; violence to animals ; disablement ; murder etc. Most studies on domestic violence can only ever approximate the extent of the issue. Statistical information can be difficult to obtain as domestic violence is often under-reported [13-23]. In Australian survey research, women are three times more likely than men to experience an episode of physical violence by their partners $[7,9,14,18]$.

To date there has been limited research on domestic violence against women with disabilities. Present research therefore focuses on various challenges faced by women with disabilities and also domestic violence and thereafter study the inter-relationship amongst them using ISM methodology . Research paper is arranged as follows : Section 2 presents the literature review. Section 3 the ISM methodology and section 4 the case example. Section 5 deals with societal implications of the problem.

\section{CHALLENGES FACED BY WOMEN WITH DISABILITIES}

Some of the major challenges are as follows:

1. Women with disabilities experience marginalization and exclusion / Vulnerability factor (VF) : This makes them "more vulnerable to violence and abuse than other women". The silence of their lived experience means that the violence in their lives is largely invisible [14,16]. These factors include dependency on others for care; denial of human rights that results in perceptions of powerlessness; less risk of discovery as perceived by the perpetrator; difficulty of being believed; economic dependence; social isolation; increased risk of manipulation; increased risk of poverty and social withdrawal

2. Colonialism (Co) : Francis, J.[5] examines the lives of Canadian aboriginal women with disabilities within the context of culture, the Christianization of indigenous religion, western medicine, gender, self-image, sexuality, alcohol and abuse.

3. Challenge of emotional violence (CEV) : Agencies reported that nearly three quarters of the women who had accessed their services experienced emotional violence. Some of society's negative images and myths regarding women with disabilities increased the women's risk of being emotionally abused in their intimate relationships.

4. Intimate partner violence (IPV): Women with intellectual disabilities being physically abused by an intimate partner, often repeatedly and severely enough to require medical attention $[17,18,20]$. They also described being threatened with severe harm, controlled, insulted, and having their property taken or destroyed. 
5. Challenge of being disabled itself (CDI): Due to physical and mental abuse these women have become disabled and suffer greater levels of poverty, social isolation, discrimination, stereotyping, low self-esteem, increased mental and physical health problems, depression and suicide $[15,16]$.

6. Long-time abuse (LTA): Women with disabilities tend to experience abuse for longer periods of time and have fewer escape options because of their greater economic dependence, their need for assistance, environmental barriers and social isolation $[4,7]$.

7. Ill equipped to handle disabilities / Lack of knowledge and education (LKE) : As per [15,16], women with disabilities are often over-protected by their relatives and carers . This over-protection and compliance training may leave women ill-equipped to handle an abusive relationship and may in fact, make them more vulnerable to abuse. Gender disparities also exist in education. While the overall literacy rate for persons with disabilities is 3 percent, UNESCO estimates that it is just 1 percent for women and girls with disabilities.

8. Lack of insight into nature of abusive relationships (LoI): Many women with intellectual disability lack insight into the nature of abusive relationships and become prone to such abuse frequently. They experienced violence perpetrated by their partner, often repeatedly and severe enough to require medical attention.

9. Inequality based on race and gender (IBR/G) : Women and girls with disabilities are subjected to multiple layers of discrimination. They are members of marginalized ethnic or racial groups or part of the lesbian, gay, bisexual, transgender and intersex community.

10. Unemployment (UE): Unemployment rates are highest among women with disabilities. The United Nations estimates that 75 percent of women with disabilities are unemployed and women with disabilities who are employed often earn less than their male counterparts and women without disabilities.

11. Denial of proper healthcare (DPH): Women with disabilities are often denied reproductive available, they may not be physically accessible for women with varying types of disabilities, or healthcare providers don't know how to accommodate them.

12. Sexual abuse (SA): The women reported experiencing many different types of abuse including physical assault, threats of assault, sexual assault as well as verbal, emotional, social and financial forms of abuse. Confusing feelings were distinct for many of the women in the study, particularly where intimate partners exercised violence $[17,18,20]$.

13. Society, Politics and disability itself (CPD): Other researchers have studied violence against women with disabilities within the broader contexts of culture, politics and disability itself. Disability already positions women at great disadvantage in the social context and the impact of poverty, isolation and discrimination are all increased when a woman with a disability experiences domestic violence [15].

14. Cultural values and religious beliefs (CVRB) : These factors can also militate against recognition of violence. If the beliefs and practices of a cultural or religious group devalue women and confine them to stereotyped and subservient roles, they can be made more vulnerable to domestic violence and accept it as an inevitable aspect of being a woman [2]. If a woman from these backgrounds also has a disability she will be doubly disadvantaged.

15. Political context (PC): Ryan evaluates the status of women with disabilities in a political context, in matters of equity and access (both physical and attitudinal), selfdetermination and the achievement of leadership position in the community. She argues that the burden of poverty prevents women with disabilities from interacting in the community [13].

16. Lack of access to formal services (LoA): Some of the women had strong reservations about approaching domestic violence crisis services for assistance and some others are so distressed with their experiences that they would not be comfortable using the service again. This was mainly because of their belief that there was a low level of access and awareness of their needs within these services.

17. Societal Beliefs (SBe): The majority of women had also not involved the police or law courts. The major barrier to the women was the belief that because police services and the courts see domestic violence as secondary to public violence, personnel in these institutions trivialized women's experiences in insensitive and intimidating stereotypical ways.

\subsection{Handling of collected data}

Statistical data analysis is usually performed on the collected data. In the qualitative data analysis, agencies were invited to participate more fully in the research by way of undertaking a semi-structured interview. In the quantitative analysis, questionnaires are usually mailed to the prospective respondents in person or through internet via emails etc. These questionnaires can then provide the information on

$\checkmark$ accessibility of the service for women with disabilities;

$\checkmark$ number of women with disabilities who had experienced family or domestic violence accessing the service in the last two years;

$\checkmark$ number of women who had acquired a disability as a result of abuse, and the number who had a history of abuse before the onset of the disability;

$\checkmark$ number of women from a non-English speaking or indigenous background;

$\checkmark \quad$ type of violence that the women experienced;

$\checkmark$ perpetrator of the violence;

$\checkmark$ estimated period of time that the women had experienced violence;

$\checkmark \quad$ types of interventions that had occurred and level of satisfaction with these interventions;

$\checkmark$ number of women who had children who were involved in the process of intervention and what would help them to respond more effectively to the needs of women with disabilities who experienced family and domestic violence.

With the help of questionnaire responses can be obtained with respect to (1) demographic profile ; 2) education profile ; 3) professional or economic profile;4) family profile etc. ;5) respondents who experience physical violence and financial violence ; 6) perpetrator of violence : family member or outsider etc. ; 7) Period of time women with disabilities experienced family and domestic violence and from there we can segregate the responses in the form of pie charts or bar 
charts etc.

\section{INTERPRETIVE STRUCTURAL MODELLING METHODOLOGY}

Proposed by Warfield [24] in ISM methodology, the process begins with the identification of relevant elements and thereafter establishing contextual relationship amongst them . A structural self-interaction matrix is created using the VAXO concept which is then followed by creation of self-interaction matrix and initial and final reachability matrix. From the reachability matrix, the reachability set and antecedent set for each criterion is found and the intersection of the two sets gives the top element [24]. After deriving the elements at various iteration levels, they are categorised as per autonomous, dependent, driver and linkage categories. Finally, a diagraph is constructed from the canonical matrix .

Around 17 challenges have been recognized viz. Vulnerability factor (VF); Colonialism (Co); Challenge of emotional violence (CEV) ; Intimate partner violence (IPV) ; Challenge of being disabled itself (CDI); Long-time abuse (LTA); Lack of knowledge and education (LKE) ; Lack of insight into nature of abusive relationships (LoI); Inequality based on race and gender (IBR/G); Unemployment (UE) ; Denial of proper healthcare (DPH) ; Sexual abuse (SA); Society, Politics and disability itself (CPD) ; Cultural values and religious beliefs (CVRB); Political context (PC); Lack of access to formal services (LoA); Societal Beliefs (SBe). These could be studied further for the possible inter-relationships amongst them using ISM methodology .

\subsection{Construction of Structural Self - Interaction Matrix (SSIM)}

This matrix gives the pair-wise relationship between two variables i.e. $i$ and $j$ based on VAXO. SSIM has been presented below in Fig 1.

\subsection{Construction of Initial Reachability Matrix and final reachability matrix}

The SSIM has been converted in to a binary matrix called the initial reachability matrix shown in fig. 2 by substituting $\mathrm{V}, \mathrm{A}$, $\mathrm{X}, \mathrm{O}$ by 1 or 0 as per the case. After incorporating the transitivity, the final reachability matrix is shown below in the Fig

Fig 1: SSIM matrix for pair wise relationship amongst barriers

\begin{tabular}{|c|c|c|c|c|c|c|c|c|c|c|c|c|c|c|c|c|c|c|}
\hline & $\begin{array}{c}\text { Barrier } \\
\text { s }\end{array}$ & 1 & 2 & 3 & 4 & 5 & 6 & 7 & 8 & 9 & 10 & 11 & 12 & 13 & 14 & 15 & 16 & 17 \\
\hline & & VF & Co & $\begin{array}{c}\text { CE } \\
\text { V }\end{array}$ & $\begin{array}{c}\text { IP } \\
\text { V }\end{array}$ & $\begin{array}{c}\text { CD } \\
\text { I }\end{array}$ & $\begin{array}{c}\text { LT } \\
\text { A }\end{array}$ & $\begin{array}{c}\text { LK } \\
\text { E }\end{array}$ & $\begin{array}{c}\text { Lo } \\
\text { I }\end{array}$ & $\begin{array}{c}\text { IB } \\
\text { R }\end{array}$ & $\begin{array}{c}\text { U } \\
\text { E }\end{array}$ & $\begin{array}{c}\text { DP } \\
\text { H }\end{array}$ & SA & $\begin{array}{c}\text { CP } \\
\text { D }\end{array}$ & $\begin{array}{c}\text { CV } \\
\text { RB }\end{array}$ & PC & LoA & SBe \\
\hline 1 & VF & & A & A & A & V & A & A & A & A & A & A & A & A & A & A & A & A \\
\hline 3 & CEV & & & & A & V & A & A & A & A & A & A & A & A & A & A & A & A \\
\hline 4 & IPV & & & & & V & X & V & A & A & A & V & V & V & A & A & V & A \\
\hline 5 & CDI & & & & & & A & A & A & A & A & A & V & A & A & A & A & A \\
\hline 6 & LTA & & & & & & & A & A & A & A & A & A & A & A & A & A & A \\
\hline 7 & LKE & & & & & & & V & V & V & V & V & V & V & V & V & V \\
\hline 8 & LoI & & & & & & & & & V & V & V & V & V & V & V & V & V \\
\hline 9 & IBR & & & & & & & & & & V & V & V & V & V & V & V & V \\
\hline 10 & UE & & & & & & & & & & V & V & V & V & V & V & V \\
\hline 11 & DPH & & & & & & & & & & & & A & A & A & A & A & A \\
\hline 12 & SA & & & & & & & & & & & & & A & A & A & A & A \\
\hline 13 & CPD & & & & & & & & & & & & & & A & A & A & A \\
\hline 14 & CVRB & & & & & & & & & & & & & & A & A & A \\
\hline 15 & PC & & & & & & & & & & & & & & & V & V \\
\hline 16 & LoA & & & & & & & & & & & & & & & & & A \\
\hline 17 & SBe & & & & & & & & & & & & & & & \\
\hline
\end{tabular}


Fig 2: Initial reachability matrix

\begin{tabular}{|c|c|c|c|c|c|c|c|c|c|c|c|c|c|c|c|c|c|c|}
\hline & Barrier & 1 & 2 & 3 & 4 & 5 & 6 & 7 & 8 & 9 & 10 & 11 & 12 & 13 & 14 & 15 & 16 & 17 \\
\hline & & $\mathrm{VF}$ & $\mathrm{Co}$ & $\begin{array}{c}\mathrm{CE} \\
\mathrm{V}\end{array}$ & $\begin{array}{l}\text { IP } \\
V\end{array}$ & $\begin{array}{c}\text { CD } \\
\text { I }\end{array}$ & $\begin{array}{c}\mathrm{LT} \\
\mathrm{A}\end{array}$ & $\begin{array}{c}\mathrm{LK} \\
\mathrm{E}\end{array}$ & $\begin{array}{c}\text { Lo } \\
\text { I }\end{array}$ & $\begin{array}{c}\text { IB } \\
\mathrm{R}\end{array}$ & $\begin{array}{l}\mathrm{U} \\
\mathrm{E}\end{array}$ & $\begin{array}{r}\mathrm{DP} \\
\mathrm{H}\end{array}$ & $\mathrm{SA}$ & $\begin{array}{c}\text { CP } \\
\text { D }\end{array}$ & $\begin{array}{l}\mathrm{CV} \\
\mathrm{RB}\end{array}$ & PC & LoA & $\mathrm{SBe}$ \\
\hline 1 & VF & 1 & 0 & 0 & 0 & 1 & 0 & 0 & 0 & 0 & 0 & 0 & 0 & 0 & 0 & 0 & 0 & 0 \\
\hline 2 & $\mathrm{Co}$ & 1 & 1 & 1 & 1 & 1 & 1 & 1 & 1 & 1 & 1 & 1 & 1 & 1 & 1 & 1 & 1 & 1 \\
\hline 3 & $\mathrm{CEV}$ & 1 & 0 & 1 & 0 & 1 & 0 & 0 & 0 & 0 & 0 & 0 & 0 & 0 & 0 & 0 & 0 & 0 \\
\hline 4 & IPV & 1 & 0 & 1 & 1 & 1 & 1 & 1 & 0 & 0 & 0 & 1 & 1 & 1 & 0 & 0 & 1 & 0 \\
\hline 5 & CDI & 0 & 0 & 0 & 0 & 1 & 0 & 0 & 0 & 0 & 0 & 0 & 1 & 0 & 0 & 0 & 0 & 0 \\
\hline 6 & LTA & 1 & 0 & 1 & 1 & 1 & 1 & 0 & 0 & 0 & 0 & 0 & 0 & 0 & 0 & 0 & 0 & 0 \\
\hline 7 & LKE & 1 & 0 & 1 & 0 & 1 & 1 & 1 & 1 & 1 & 1 & 1 & 1 & 1 & 1 & 1 & 1 & 1 \\
\hline 8 & LoI & 1 & 0 & 1 & 1 & 1 & 1 & 0 & 1 & 1 & 1 & 1 & 1 & 1 & 1 & 1 & 1 & 1 \\
\hline 9 & IBR & 1 & 0 & 1 & 1 & 1 & 1 & 0 & 0 & 1 & 1 & 1 & 1 & 1 & 1 & 1 & 1 & 1 \\
\hline 10 & UE & 1 & 0 & 1 & 1 & 1 & 1 & 0 & 0 & 0 & 1 & 1 & 1 & 1 & 1 & 1 & 1 & 1 \\
\hline 11 & DPH & 1 & 0 & 1 & 0 & 1 & 1 & 0 & 0 & 0 & 0 & 1 & 0 & 0 & 0 & 0 & 0 & 0 \\
\hline 12 & SA & 1 & 0 & 1 & 0 & 0 & 1 & 0 & 0 & 0 & 0 & 1 & 1 & 0 & 0 & 0 & 0 & 0 \\
\hline 13 & CPD & 1 & 0 & 1 & 0 & 1 & 1 & 0 & 0 & 0 & 0 & 1 & 1 & 1 & 0 & 0 & 0 & 0 \\
\hline 14 & CVRB & 1 & 0 & 1 & 1 & 1 & 1 & 0 & 0 & 0 & 0 & 1 & 1 & 1 & 1 & 0 & 0 & 0 \\
\hline 15 & $\mathrm{PC}$ & 1 & 0 & 1 & 1 & 1 & 1 & 0 & 0 & 0 & 0 & 1 & 1 & 1 & 1 & 1 & 1 & 1 \\
\hline 16 & LoA & 1 & 0 & 1 & 1 & 1 & 1 & 0 & 0 & 0 & 0 & 1 & 1 & 1 & 1 & 0 & 1 & 0 \\
\hline 17 & $\mathrm{SBe}$ & 1 & 0 & 1 & 1 & 1 & 1 & 0 & 0 & 0 & 0 & 1 & 1 & 1 & 1 & 0 & 1 & 1 \\
\hline
\end{tabular}

Fig 3 : Final reachability matrix

\begin{tabular}{|c|c|c|c|c|c|c|c|c|c|c|c|c|c|c|c|c|c|c|c|}
\hline & Barrier & 1 & 2 & 3 & 4 & 5 & 6 & 7 & 8 & 9 & 10 & 11 & 12 & 13 & 14 & 15 & 16 & 17 & D.P \\
\hline & & VF & $\mathrm{Co}$ & $\begin{array}{c}\mathrm{CE} \\
\mathrm{V}\end{array}$ & $\begin{array}{l}\text { IP } \\
V\end{array}$ & $\begin{array}{c}\text { CD } \\
\text { I }\end{array}$ & $\begin{array}{c}\mathrm{LT} \\
\mathrm{A}\end{array}$ & $\begin{array}{c}\text { LK } \\
\text { E }\end{array}$ & $\begin{array}{c}\text { Lo } \\
\text { I }\end{array}$ & $\begin{array}{c}\text { IB } \\
\mathrm{R}\end{array}$ & $\begin{array}{l}\mathrm{U} \\
\mathrm{E}\end{array}$ & $\begin{array}{c}\text { DP } \\
\mathrm{H}\end{array}$ & SA & $\begin{array}{c}\text { CP } \\
D\end{array}$ & $\begin{array}{l}\text { CV } \\
\text { RB }\end{array}$ & $\mathrm{PC}$ & LoA & $\mathrm{SBe}$ & \\
\hline 1 & VF & 1 & 0 & 0 & 0 & 1 & 0 & 0 & 0 & 0 & 0 & 0 & 1 & 0 & 0 & 0 & 0 & 0 & 3 \\
\hline 2 & $\mathrm{Co}$ & 1 & 1 & 1 & 1 & 1 & 1 & 1 & 1 & 1 & 1 & 1 & 1 & 1 & 1 & 1 & 1 & 1 & 17 \\
\hline 3 & CEV & 1 & 0 & 1 & 0 & 1 & 0 & 0 & 0 & 0 & 0 & 0 & 1 & 0 & 0 & 0 & 0 & 0 & 3 \\
\hline 4 & IPV & 1 & 0 & 1 & 1 & 1 & 1 & 0 & 1 & 1 & 1 & 1 & 1 & 1 & 1 & 1 & 1 & 1 & 15 \\
\hline 5 & CDI & 1 & 0 & 1 & 0 & 1 & 1 & 0 & 0 & 0 & 0 & 1 & 1 & 0 & 0 & 0 & 0 & 0 & 6 \\
\hline 6 & LTA & 1 & 0 & 1 & 1 & 1 & 1 & 1 & 0 & 0 & 0 & 1 & 1 & 1 & 0 & 0 & 0 & 0 & 9 \\
\hline 7 & LKE & 1 & 0 & 1 & 1 & 1 & 1 & 1 & 1 & 1 & 1 & 1 & 1 & 1 & 1 & 1 & 1 & 1 & 16 \\
\hline 8 & LoI & 1 & 0 & 1 & 1 & 1 & 1 & 0 & 1 & 1 & 1 & 1 & 1 & 1 & 1 & 1 & 1 & 1 & 15 \\
\hline 9 & IBR & 1 & 0 & 1 & 1 & 1 & 1 & 0 & 0 & 1 & 1 & 1 & 1 & 1 & 1 & 1 & 1 & 1 & 13 \\
\hline 10 & UE & 1 & 0 & 1 & 1 & 1 & 1 & 0 & 0 & 0 & 1 & 1 & 1 & 1 & 1 & 1 & 1 & 1 & 13 \\
\hline 11 & $\mathrm{DPH}$ & 1 & 0 & 1 & 1 & 1 & 1 & 0 & 0 & 0 & 0 & 1 & 1 & 0 & 0 & 0 & 0 & 0 & 6 \\
\hline 12 & SA & 1 & 0 & 1 & 0 & 1 & 1 & 0 & 0 & 0 & 0 & 1 & 1 & 0 & 0 & 0 & 0 & 0 & 6 \\
\hline 13 & CPD & 1 & 0 & 1 & 0 & 1 & 1 & 0 & 0 & 1 & 0 & 1 & 1 & 1 & 1 & 1 & 1 & 1 & 12 \\
\hline 14 & CVRB & 1 & 0 & 1 & 1 & 1 & 1 & 0 & 0 & 0 & 0 & 1 & 1 & 1 & 1 & 0 & 0 & 0 & 9 \\
\hline
\end{tabular}




\begin{tabular}{|c|c|c|c|c|c|c|c|c|c|c|c|c|c|c|c|c|c|c|c|}
\hline 15 & PC & 1 & 0 & 1 & 1 & 1 & 1 & 0 & 0 & 0 & 0 & 1 & 1 & 1 & 1 & 1 & 1 & 1 & 12 \\
\hline 16 & LoA & 1 & 0 & 1 & 1 & 1 & 1 & 0 & 0 & 0 & 0 & 1 & 1 & 1 & 1 & 0 & 1 & 0 & 10 \\
\hline 17 & SBe & 1 & 0 & 1 & 1 & 1 & 1 & 0 & 0 & 0 & 0 & 1 & 1 & 1 & 1 & 0 & 1 & 1 & 11 \\
\hline & De.P & 17 & 1 & 16 & 12 & 17 & 15 & 3 & 4 & 6 & 6 & 15 & 17 & 12 & 11 & 8 & 10 & 9 & \\
\hline
\end{tabular}

D.P : Driving power ; De.P : dependence power

\subsection{Level Partition}

From the final reachability matrix, reachability and final antecedent set for each factor are found. The elements for which the reachability and intersection sets are same are the top-level element in the ISM hierarchy. After the identification of top level element, it is separated out from the other elements and the process continues for next level of elements. Reachability set, antecedent set, intersection set along with different level for elements have been shown below in table 1 to table 6 .

Table 1: Iteration I

\begin{tabular}{|c|c|c|c|c|}
\hline $\begin{array}{c}\text { S. } \\
\text { No } \\
\text {. }\end{array}$ & Reachability set & Antecedent set & $\begin{array}{c}\text { Inters } \\
\text { ection } \\
\text { set }\end{array}$ & $\begin{array}{l}\text { Le } \\
\text { vel }\end{array}$ \\
\hline 1. & $1,5,12$ & $\begin{array}{c}1,2,3,4,5,6,7,8 \\
9,10 \\
11,12,13,14,15 \\
16,17\end{array}$ & $1,5,12$ & \multirow{4}{*}{ I } \\
\hline 2. & $1,3,5,12$ & $\begin{array}{c}2,3,4,5,6,7,8,9, \\
10,11, \\
12,13,14,15,16, \\
17\end{array}$ & $3,5,12$ & \\
\hline 3. & $1,3,5,6,11,12$ & $\begin{array}{c}2,4,5,6,7,8,9,10 \\
, 11,12, \\
13,14,15,16,17\end{array}$ & $\begin{array}{c}1,5,6,1 \\
1,12\end{array}$ & \\
\hline 4. & $1,3,4,5,6,11,12$ & $2,4,6,7,8,9,10,1$ & $4,6,11$ & \\
\hline
\end{tabular}

\begin{tabular}{|c|c|c|c|}
\hline & & $1,12,13$, & \\
& & $14,15,16,17$ & \\
\hline 5. & $1,3,4,5,6,11,12,13$ & $2,4,6,7,8,9,10,1$ & $4,6,13$ \\
& & $3,14,15,16,17$ & \\
\hline 6. & $1,3,4,5,6,11,12,13$, & $2,4,7,8,9,10,14$, & 4,14 \\
& 14 & $15,16,17$ & \\
\hline 7. & $1,3,4,5,6,11,12,13$, & $2,4,7,8,9,10,15$, & 4,16 \\
& 14,16 & 16,17 & \\
\hline 8 & $1,3,4,5,6,11,12,13$, & $2,4,7,8,9,10,15$, & 4,17 \\
& $14,16,17$ & 17 & \\
\hline 9 & $1,3,4,5,6,11,12,13$, & $2,4,7,8,9,10,15$ & 4,15 \\
& $14,15,16,17$ & & \\
\hline 10 & $1,3,4,5,6,9,10,11,12$, & $2,4,7,8,9,10,13$ & $4,9,13$ \\
$\cdot$ & $13,14,15,16,17$ & & \\
\hline 11 & $1,3,4,5,6,8,9,10,11$, & $2,4,7,8$ & 4,8 \\
$\cdot$ & $12,13,14,15,16,17$ & & \\
\hline 12 & $1,3,4,5,6,7,8,9,10,11$, & $2,7,8$ & 7,8 \\
$\cdot$ & $12,13,14,15,16,17$ & & \\
\hline 13 & $1,2,3,4,5,6,7,8,9,10,1$ & & \\
$\cdot$ & $1,12,13,14,15,16$, & & \\
& 17 & & \\
\hline
\end{tabular}

Table 2: Iteration II

\begin{tabular}{|c|c|c|c|c|}
\hline S.No & Reachability set & Antecedent set & Intersection set & Level \\
\hline 2. & 3 & $2,3,4,6,7,8,9,10,11,13,14,15,16,17$ & 3 & \multirow{8}{*}{ II } \\
\hline 3. & $1,3,5,6,11,12$ & $2,4,5,6,7,8,9,10,11,12,13,14,15,16,17$ & $1,5,6,11,12$ & \\
\hline 4. & $1,3,4,5,6,11,12$ & $2,4,6,7,8,9,10,11,12,13,14,15,16,17$ & $4,6,11$ & \\
\hline 5. & $1,3,4,5,6,11,12,13$ & $\begin{array}{c}2,4,6,7,8,9,10,13 \\
14,15,16,17\end{array}$ & $4,6,13$ & \\
\hline 6. & $\begin{array}{c}1,3,4,5,6,11,12,13 \\
14\end{array}$ & $2,4,7,8,9,10,14,15,16,17$ & 4,14 & \\
\hline 7. & $\begin{array}{c}1,3,4,5,6,11,12,13 \\
14,16\end{array}$ & $2,4,7,8,9,10,15,16,17$ & 4,16 & \\
\hline 8. & $\begin{array}{c}1,3,4,5,6,11,12,13 \\
14,16,17\end{array}$ & $2,4,7,8,9,10,15,17$ & 4,17 & \\
\hline 9. & $1,3,4,5,6,11,12,13$ & $2,4,7,8,9,10,15$ & 4,15 & \\
\hline
\end{tabular}




\begin{tabular}{|c|c|c|c|}
\hline & $14,15,16,17$ & & \\
\hline 10. & $1,3,4,5,6,9,10,11$, & $2,4,7,8,9,10,13$ & $4,9,13$ \\
& $12,13,14,15,16,17$ & $2,4,7,8$ & 4,8 \\
\hline 11. & $1,3,4,5,6,8,9,10,11,12,13,14,15,16,17$ & $2,7,8$ & 7,8 \\
\hline 12. & $1,3,4,5,6,7,8,9,10,11,12,13,14,15,16,17$ & 2 & 2 \\
\hline 13. & $1,2,3,4,5,6,7,8,9$, & & \\
\hline
\end{tabular}

Table 3 : Iteration III

\begin{tabular}{|c|c|c|c|c|}
\hline $\begin{array}{l}\text { S. } \\
\text { No. }\end{array}$ & $\begin{array}{c}\text { Reachability } \\
\text { set }\end{array}$ & $\begin{array}{c}\text { Antecedent } \\
\text { set }\end{array}$ & $\begin{array}{c}\text { Intersection } \\
\text { set }\end{array}$ & Level \\
\hline 3. & 6,11 & $\begin{array}{c}2,4,6,7,8,9,10, \\
11,13,14,15,16 \\
, 17\end{array}$ & 6,11 & III \\
\hline 4. & $\begin{array}{c}1,3,4,5,6,11 \\
12\end{array}$ & $\begin{array}{c}2,4,6,7,8,9,10, \\
11,12,13,14,15 \\
, 16,17\end{array}$ & $4,6,11$ & \\
\hline 5. & $\begin{array}{c}1,3,4,5,6,11 \\
12,13\end{array}$ & $\begin{array}{c}2,4,6,7,8,9,10, \\
13,14,15,16,17\end{array}$ & $4,6,13$ & \\
\hline 6. & $\begin{array}{c}1,3,4,5,6,11 \\
12,13,14\end{array}$ & $\begin{array}{c}2,4,7,8,9,10,14 \\
, 15,16,17\end{array}$ & 4,14 & \\
\hline 7. & $\begin{array}{l}1,3,4,5,6,11 \\
12,13,14,16\end{array}$ & $\begin{array}{c}2,4,7,8,9,10,15 \\
, 16,17\end{array}$ & 4,16 & \\
\hline 8. & $\begin{array}{c}1,3,4,5,6,11 \\
12,13,14,16 \\
17\end{array}$ & $\begin{array}{c}2,4,7,8,9,10,15 \\
, 17\end{array}$ & 4,17 & \\
\hline 9. & $\begin{array}{c}1,3,4,5,6,11 \\
12,13,14,15 \\
16,17\end{array}$ & $2,4,7,8,9,10,15$ & 4,15 & \\
\hline 10. & $\begin{array}{c}1,3,4,5,6,9,10,1 \\
1,12,13,14, \\
15,16,17\end{array}$ & $2,4,7,8,9,10,13$ & $4,9,13$ & \\
\hline 11. & $\begin{array}{c}1,3,4,5,6,8,9 \\
10 \\
11,12,13,14 \\
15,16,17\end{array}$ & $2,4,7,8$ & 4,8 & \\
\hline 12. & $\begin{array}{c}1,3,4,5,6,7,8,9 \\
10,11,12,13 \\
14,15,16,17\end{array}$ & $2,7,8$ & 7,8 & \\
\hline 13. & $\begin{array}{c}1,2,3,4,5,6,7,8 \\
9,10,11,12,13,1 \\
4,15,16,17\end{array}$ & 2 & 2 & \\
\hline
\end{tabular}

Table 4 : Iteration IV

\begin{tabular}{|c|c|c|c|c|}
\hline $\begin{array}{l}\text { S. } \\
\text { No. }\end{array}$ & Reachability set & Antecedent set & $\begin{array}{c}\text { Interse } \\
\text { ction } \\
\text { set }\end{array}$ & $\begin{array}{l}\text { Le } \\
\text { vel }\end{array}$ \\
\hline 4. & 4 & $\begin{array}{c}2,4,7,8,9,10,13,1 \\
4,15,16,17\end{array}$ & 4 & \multirow{10}{*}{ IV } \\
\hline 5. & $\begin{array}{c}1,3,4,5,6,11,12, \\
13\end{array}$ & $\begin{array}{l}2,4,6,7,8,9,10, \\
13,14,15,16,17\end{array}$ & $4,6,13$ & \\
\hline 6. & $\begin{array}{c}1,3,4,5,6,11,12, \\
13,14\end{array}$ & $\begin{array}{c}2,4,7,8,9,10,14,1 \\
5,16,17\end{array}$ & 4,14 & \\
\hline 7. & $\begin{array}{c}1,3,4,5,6,11,12, \\
13,14,16\end{array}$ & $\begin{array}{c}2,4,7,8,9,10,15,1 \\
6,17\end{array}$ & 4,16 & \\
\hline 8. & $\begin{array}{c}1,3,4,5,6,11,12, \\
13,14,16,17\end{array}$ & $\begin{array}{c}2,4,7,8,9,10,15,1 \\
7\end{array}$ & 4,17 & \\
\hline 9. & $\begin{array}{l}1,3,4,5,6,11,12, \\
13,14,15,16,17\end{array}$ & $2,4,7,8,9,10,15$ & 4,15 & \\
\hline 10. & $\begin{array}{c}1,3,4,5,6,9,10,11,12 \\
13,14,15,16,17\end{array}$ & $2,4,7,8,9,10,13$ & $4,9,13$ & \\
\hline 11. & $\begin{array}{c}1,3,4,5,6,8,9,10,11 \\
12,13,14,15,16,17\end{array}$ & $2,4,7,8$ & 4,8 & \\
\hline 12. & $\begin{array}{c}1,3,4,5,6,7,8,9,10,11,1 \\
2,13,14,15,16,17\end{array}$ & $2,7,8$ & 7,8 & \\
\hline 13. & $\begin{array}{c}1,2,3,4,5,6,7,8,9,10, \\
11,12,13,14,15,16,17\end{array}$ & 2 & 2 & \\
\hline
\end{tabular}

Table 5: Iteration V

\begin{tabular}{|c|c|c|c|c|}
\hline $\begin{array}{c}\text { S. } \\
\text { No } \\
\cdot\end{array}$ & Reachability set & Antecedent set & $\begin{array}{c}\text { Intersecti } \\
\text { on set }\end{array}$ & $\begin{array}{c}\text { Le } \\
\text { vel }\end{array}$ \\
\hline 5. & $\mathbf{1 3}$ & $\begin{array}{c}2,4,6,7,8,9,10,13,1 \\
4,15,16,17\end{array}$ & 13 & \\
\hline 6. & $\begin{array}{c}1,3,4,5,6,11,12,1 \\
3,14\end{array}$ & $\begin{array}{c}2,4,7,8,9,10,14,15, \\
16,17\end{array}$ & 4,14 & \\
\cline { 1 - 4 } 7. & $\begin{array}{c}1,3,4,5,6,11,12,1 \\
\end{array}$ & $\begin{array}{c}2,4,7,8,9,10,15,16, \\
17\end{array}$ & 4,16 & \\
\hline 8. & $\begin{array}{c}1,3,4,5,6,11,12,1 \\
3,14,16,17\end{array}$ & $2,4,7,8,9,10,15,17$ & 4,17 & \\
\hline
\end{tabular}




\begin{tabular}{|c|c|c|c|}
\hline 9. & $\begin{array}{c}1,3,4,5,6,11,12,1 \\
3,14,15,16,17\end{array}$ & $2,4,7,8,9,10,15$ & 4,15 \\
\hline 10. & $\begin{array}{c}1,3,4,5,6,9,10 \\
11,12,13,14,15,1 \\
6,17\end{array}$ & $2,4,7,8,9,10,13$ & $4,9,13$ \\
\hline 11. & $\begin{array}{c}1,3,4,5,6,8,9,10,1 \\
1,12,13,14,15, \\
16,17\end{array}$ & $2,4,7,8$ & 4,8 \\
\hline 12. & $\begin{array}{c}1,3,4,5,6,7,8,9 \\
10,11,12,13,14,1 \\
5,16,17\end{array}$ & $2,7,8$ & 7,8 \\
\hline 13. & $\begin{array}{c}1,2,3,4,5,6,7,8,9 \\
10,11,12,13,14 \\
15,16,17\end{array}$ & 2 & 2 \\
\hline
\end{tabular}

Table 6: Iteration VI

\begin{tabular}{|c|c|c|c|c|}
\hline $\begin{array}{l}\text { S. } \\
\text { N } \\
\text { o. }\end{array}$ & $\begin{array}{c}\text { Reachability } \\
\text { set }\end{array}$ & Antecedent set & $\begin{array}{c}\text { Intersec } \\
\text { tion set }\end{array}$ & $\begin{array}{c}\text { Lev } \\
\text { el }\end{array}$ \\
\hline 6. & 4,14 & $\begin{array}{c}2,4,7,8,9,10,14,1 \\
5,16,17\end{array}$ & 4,14 & \\
\hline 7. & $4,14,16$ & $\begin{array}{c}2,4,7,8,9,10,15,1 \\
6,17\end{array}$ & 4,16 & \\
\hline 8. & $4,14,16,17$ & $\begin{array}{c}2,4,7,8,9,10,15,1 \\
7\end{array}$ & 4,17 & \\
\hline 9. & $4,14,15,16,17$ & $2,4,7,8,9,10,15$ & 4,15 & VI \\
\hline $\begin{array}{c}10 \\
.\end{array}$ & $\begin{array}{c}4,9,10,14,15,16 \\
17\end{array}$ & $2,4,7,8,9,10$ & 4,9 & \\
\hline 11 & $\begin{array}{c}4,8,9,10,14,15 \\
16,17\end{array}$ & $2,4,7,8$ & 4,8 & \\
\hline 12 & $\begin{array}{c}4,7,8,9,10,14,15 \\
, 16,17\end{array}$ & $2,7,8$ & 7,8 & \\
\hline 13 & $\begin{array}{c}2,4,7,8,9,10,14 \\
15,16,17\end{array}$ & 2 & 2 & \\
\hline
\end{tabular}

Table 7: Iteration VII

\begin{tabular}{|c|c|c|c|c|}
\hline $\begin{array}{l}\text { S. } \\
\text { No } \\
\text {. }\end{array}$ & $\begin{array}{c}\text { Reachabilit } \\
\text { y set }\end{array}$ & Antecedent set & $\begin{array}{c}\text { Intersectio } \\
\text { n set }\end{array}$ & $\begin{array}{c}\text { Leve } \\
1\end{array}$ \\
\hline 7. & 16 & $\begin{array}{c}2,7,8,9,10,15,1 \\
6,17\end{array}$ & 16 & \multirow{6}{*}{ VII } \\
\hline 8. & 16,17 & $\begin{array}{c}2,7,8,9,10,15,1 \\
7\end{array}$ & 17 & \\
\hline 9. & $15,16,17$ & $2,7,8,9,10,15$ & 15 & \\
\hline 10. & $\begin{array}{c}9,10,15,16, \\
17\end{array}$ & $2,7,8,9,10$ & 9 & \\
\hline 11. & $\begin{array}{c}8,9,10,15,16 \\
17\end{array}$ & $2,7,8$ & 8 & \\
\hline 12. & $7,8,9,10,15$ & $2,7,8$ & 7,8 & \\
\hline
\end{tabular}

\begin{tabular}{|c|c|c|c|c|}
\hline & 16,17 & & & \\
\hline 13. & $\begin{array}{c}2,7,8,9,10,15 \\
16,17\end{array}$ & 2 & 2 & \\
\hline
\end{tabular}

Table 8: Iteration VIII

\begin{tabular}{|c|c|c|c|c|}
\hline $\begin{array}{l}\text { S. } \\
\text { No }\end{array}$ & $\begin{array}{c}\text { Reachability } \\
\text { set }\end{array}$ & $\begin{array}{c}\text { Antecedent } \\
\text { set }\end{array}$ & $\begin{array}{c}\text { Intersectio } \\
\text { n set }\end{array}$ & $\begin{array}{c}\text { Leve } \\
1\end{array}$ \\
\hline 8. & 17 & $\begin{array}{c}2,7,8,9,10,15,1 \\
7\end{array}$ & 17 & \multirow{6}{*}{ VIII } \\
\hline 9. & 15,17 & $2,7,8,9,10,15$ & 15 & \\
\hline 10 . & $9,10,15,17$ & $2,7,8,9,10$ & 9 & \\
\hline 11. & $\begin{array}{c}8,9,10,14,15,1 \\
7\end{array}$ & $2,7,8$ & 8 & \\
\hline 12 . & $\begin{array}{c}7,8,9,10,14 \\
15,17\end{array}$ & $2,7,8$ & 7,8 & \\
\hline 13. & $\begin{array}{c}2,7,8,9,10,14 \\
15,17\end{array}$ & 2 & 2 & \\
\hline
\end{tabular}

Table 9: Iteration IX

\begin{tabular}{|c|c|c|c|c|}
\hline $\begin{array}{c}\text { S. } \\
\text { No. }\end{array}$ & $\begin{array}{c}\text { Reachability } \\
\text { set }\end{array}$ & $\begin{array}{c}\text { Antecedent } \\
\text { set }\end{array}$ & $\begin{array}{c}\text { Intersection } \\
\text { set }\end{array}$ & Level \\
\cline { 1 - 3 } 9. & $\mathbf{1 5}$ & $2,7,8,9,10,15$ & 15 & \\
\hline 10. & $9,10,15$ & $2,7,8,9,10$ & 9 & \\
\hline 11. & $8,9,10,15$ & $2,7,8$ & 8 & \multirow{2}{*}{ IX } \\
\cline { 1 - 3 } 12. & $7,8,9,10,15$ & $2,7,8$ & 7,8 & \\
\hline 13. & $2,7,8,9,10,15$ & 2 & 2 & \\
\hline
\end{tabular}

Table 10: Iteration $X$

\begin{tabular}{|c|c|c|c|c|}
\hline $\begin{array}{c}\text { S. } \\
\text { No. }\end{array}$ & $\begin{array}{c}\text { Reachability } \\
\text { set }\end{array}$ & $\begin{array}{c}\text { Antecedent } \\
\text { set }\end{array}$ & $\begin{array}{c}\text { Intersection } \\
\text { set }\end{array}$ & Level \\
\cline { 1 - 4 } 10. & $\mathbf{9 , 1 0}$ & $2,7,8,9,10$ & $\mathbf{9 , 1 0}$ & \multirow{2}{*}{$\mathbf{X}$} \\
\cline { 1 - 4 } 11. & $8,9,10$ & $2,7,8$ & 8 & \\
\cline { 1 - 3 } 12. & $7,8,9,10$ & $2,7,8$ & 7,8 & \\
\hline 13. & $2,7,8,9,10$ & 2 & 2 & \\
\hline
\end{tabular}

Table 11: Iteration XI

\begin{tabular}{|c|c|c|c|c|}
\hline $\begin{array}{c}\text { S. } \\
\text { No. }\end{array}$ & $\begin{array}{c}\text { Reachability } \\
\text { set }\end{array}$ & $\begin{array}{c}\text { Antecedent } \\
\text { set }\end{array}$ & $\begin{array}{c}\text { Intersection } \\
\text { set }\end{array}$ & Level \\
\cline { 1 - 4 } 11. & $\mathbf{8}$ & $2,7,8$ & 8 & \multirow{2}{*}{ XI } \\
\cline { 1 - 3 } 12. & 7,8 & $2,7,8$ & 7,8 & \\
\hline 13. & $2,7,8$ & 2 & 2 & \\
\hline
\end{tabular}


Table 12: Iteration XII

\begin{tabular}{|c|c|c|c|c|}
\hline $\begin{array}{c}\text { S. } \\
\text { No. }\end{array}$ & $\begin{array}{c}\text { Reachability } \\
\text { set }\end{array}$ & $\begin{array}{c}\text { Antecedent } \\
\text { set }\end{array}$ & $\begin{array}{c}\text { Intersection } \\
\text { set }\end{array}$ & Level \\
\hline 12. & $\mathbf{7}$ & 2,7 & 7 & XII \\
\hline 13. & 2,7, & 2 & 2 & \\
\hline
\end{tabular}

Table 13: Iteration XIII

\begin{tabular}{|c|c|c|c|c|}
\hline $\begin{array}{c}\text { S. } \\
\text { No. }\end{array}$ & $\begin{array}{c}\text { Reachability } \\
\text { set }\end{array}$ & $\begin{array}{c}\text { Antecedent } \\
\text { set }\end{array}$ & $\begin{array}{c}\text { Intersection } \\
\text { set }\end{array}$ & Level \\
\hline 13. & 2 & 2 & 2 & XIII \\
\hline
\end{tabular}

\subsubsection{Classification of factors}

The critical success factors described earlier are classified in to four clusters viz. autonomous factor, dependent factors, linkage factors and independent / Driving factors are mentioned below.

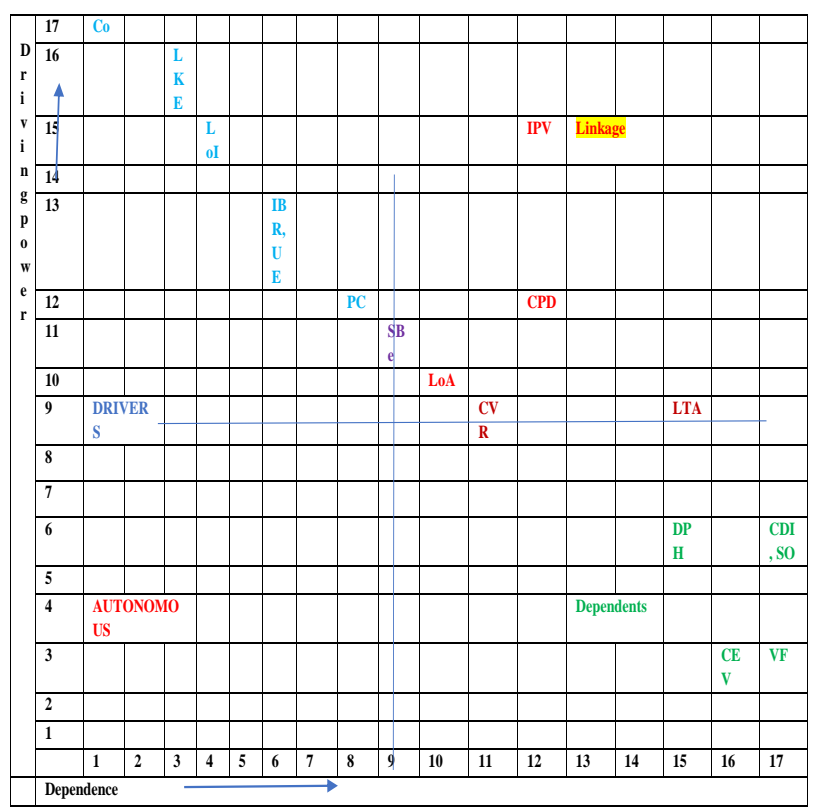

\section{LITERARY OBSERVATIONS AND SOCIETAL IMPLICATIONS \\ 5.1Special actions taken by different agencies}

- Specialised counselling services for women with disabilities.

- Earlier intervention and education in protective behaviours and relationships to shift the attitudes of women themselves.

- The needs of children of women with a disability experiencing domestic violence.

- Recognition of the increasing prevalence of financial abuse experienced by women with disabilities.

- Understanding the difficulties in speaking out experienced by women with disabilities (especially older women) in small towns, where support services may be in short supply.

- Recognition of the basic human and legal rights of women with disabilities to make their own choices.

- Awareness and understanding of cross-cultural issues as they affect women with disabilities.

- Services and assistance structured to empower women to make changes for themselves.

- More awareness that many women with disabilities are subjected to predatory behaviour because of their vulnerabilities.

- More funding for education programmes.

\subsection{Societal implications}

1. The exclusion and violence against women and girls with disabilities in any country carries heavy financial and social consequences. Discrimination against persons with disabilities hinders economic development, limits democracy, and erodes societies.

2. Perhaps because of the challenges they face, women and girls with disabilities are poised to be leaders within their communities and can greatly contribute to the economic development of their countries.

3. Women from marginalised groups often resist mistreatment . Many of the women blamed themselves and expected less for themselves in the context of the real difficulties they found themselves in

\section{ACKNOWLEDGMENTS}

Authors pays their sincere regards and big thanks to the women who shared their stories about their experiences with family and domestic violence in the hope that they would help other women in similar circumstances. Our thanks to the anonymous reviewers whose comments have helped us in improving the manuscript .

\section{REFERENCES}

[1] Boyle, G., Rioux, M., Ticoll, M. \& Wright-Felske, A. 1988. Women with disabilities: A national forum. Entourage, 9-14.

[2] Buchmeuller, K. 1996. Women with Disabilities and Domestic Violence: Issues and strategies. Brisbane: Queensland Women's Consultative Council.

[3] Carlson, B. 1998. Domestic violence in adults with mental retardation: Reports from victims and key informants. Mental Health Aspects of Developmental disabilities. 1(4), 102-112.

[4] Centre for Research on Women With Disabilities 1999. National Study of Women with Physical Disabilities:

[5] Francis, J. 1992. Voices in the Wilderness: Aboriginal Women and Disabilities. National Aboriginal Network on Disability, Ottawa.

[6] Gilson, S. F., Cramer, E. P., \& DePoy, E. 2001. Redefining Abuse of Women with Disabilities: A Paradox of Limitation and Expansion. AFFILIA, 16(2), 220-235.

[7] Pane, L. 1994. Emerging From the Shadows: A Report of 
the Status of Women with Disabilities Living in Australia. Curtin, ACT, Australia: Women With Disabilities Australia. Retrieved, from the World Wide Web:

[8] Ryan, C. 2002. An Inclusive Democracy? Paper presented at the Women's Constitutional Convention 2002 "Trust The Women". Women With Disabilities, Canberra, ACT.

[9] Sceriha, M. 1996. Women With Disabilities and Domestic Violence. Women with Disabilities Australia. Retrieved 7th April 2003, from the World Wide Web: www.wwda.org.au/madge.htm

[10] Francis, J. 1992. Voices in the Wilderness: Aboriginal Women and Disabilities. National Aboriginal Network on Disability, Ottawa. Retrieved 18 March 2003, from the World Wide Web: www.schoolnet.ca/aboroginal/disabl6/acknow-e.html

[11] Schaller, J. \& Fieberg, J.L. 1998. Issues of abuse for women with disabilities and implications for rehabilitation counselling. Journal of Applied Rehabilitation Counselling, (29), 9-17.

[12] Hassounch-Phillips, D., \& Curry, M. A. 2002. Abuse of Women With Disabilities: State of the Science. Rehabilitation Counseling Bulletin, 45(2), 96-104.

[13] Ryan, C. 2002. Women With Disabilities ACT: An Inclusive Democracy? Paper presented at the Women's Constitutional Convention 2002 "Trust The Women".

[14] Focht-New, V. 1997. Beyond Abuse: Treatment Approaches for People with Disabilities. 1(2), 212-217.

[15] Curry, M. A., Hassounch-Phillips, D., \& JohnstonSilverberg, A. 2001. Abuse of Women With Disabilities: An Ecological Model and Review Violence Against Women 7(1).
[16] Gilson, S. F., DePoy, E., \& Cramer, E. P. 2001. Linking the Assessment of Self-Reported Functional Capacity With Abuse Experiences of Women With Disabilities. Violence Against Women, 7(4), 418-431.

[17] Alexander, R. 1993. Wife-battering-an Australian perspective. Journal of Family Violence (8) 229-251.

[18] Bagshaw, D. \& Chung, D. 2000. Women, men and domestic violence.

[19] De Vries, R.M., March, L. \& Vinen J. 1996. Prevalence of domestic violence among patients attending a hospital emergency department. Australian \& New Zealand Journal of Public Health. (20). 364-368.

[20] Hegarty, K. \& Roberts, G. 1998. How common is domestic violence against women? The defintion of partner abuse in prevalence studies. Australian \& New Zealand Journal of Public Health, (22), 49-54.

[21] McMullen O'Brien, S. 2002. Staying Alive: A Client With Chronic Mental Illness in an Environment of Domestic Violence http://proquest.umi.com/pqdweb?Did=00000012202783 $\&$ Fmt $=3 \&$ Deli $=1 \& \mathrm{Mtd}=1 \& \mathrm{Idx}=18 \& \mathrm{Sid}=1 \& \mathrm{RQT}=309$

[22] Diaz-Olavarrieta, C., Campbell, J., Garcia de la Cadena, C., Paz, F., \& E Villa, A. 1999. Domestic Violence Against Patients With Chronic Neurologic Disorders Archives of Neurology, Chicago, June 1999 56 (6) 321-328.

[23] McMullen O’Brien, S. 2002. Staying Alive: A Client With Chronic Mental Illness in an Environment of Domestic Violence [Online Journal: Holistic Nursing Practice 16 (3), pp. 16-23.

[24] Warfield, J. N. 1974. Developing interconnection matrices in structural modeling. IEEE Transactions on System, Man, and Cybernetics, SMC-4 (1), 81-87. 